\title{
Comparison of Tumor Volume Dependent Mean Diameter with the Longest Diameter and Tumor Volume in Assessing Axillary Lymph Nodes in Breast Cancer
}

\author{
Mehmet Kubat and Zülfikar Karabulut \\ Department of General Surgery, ALKU Alanya Training and Research Hospital, Alanya, Turkey
}

\begin{abstract}
Objective: To investigate the effectiveness of tumor volume dependent mean diameter (Rmax) in determining axillary lymph node metastasis positivity instead of the largest diameter (Dmax) in breast cancer.

Study Design: Descriptive study.

Place and Duration of Study: Department of General Surgery, Ankara Training and Reseach Hospital, Turkey, from January 2009 to December 2018.

Methodology: Patients with breast cancer, who underwent modified radical mastectomy and lumpectomy and axillary lymph node dissection due to invasive carcinoma, included in this study. Approximate vTm was calculated from the obtained tumor specimen dimensions using ellipsoid volume formula. From the sphere volume formula, Rmax, which gives the same vTm value, was reversely calculated. Efficacy and usability of Rmax value were evaluated on nomograms that are available online; and are used most frequently owing to its ease of use.

Results: In 305 patients, mean Dmax was $3.4 \pm 1.8 \mathrm{~cm}$, mean vTm was $17.9 \pm 35.9 \mathrm{~cm}^{3}$, and mean Rmax was $2.6 \pm 1.4 \mathrm{~cm}$. For the distinction of ALNM positive and negative patients, Dmax significant [Area Under Curve (AUC) 0.709], Rmax significant [AUC 0.748] and vTm significant [AUC 0.748] efficacy was considered.

Conclusion: When the relationships of Dmax and VTm with ALNM were compared in breast cancer cases, the efficacy of vTm is higher. It was found that vTm and Rmax can be used instead of Dmax. The relation of Rmax parameter with ALNM was found to be higher. When Rmax was used in nomogram samples, which are currently used in predicting ALNM positivity, it increased nomogram efficacy.
\end{abstract}

Key Words: Breast neoplasm, Tumor burden, Tumor volume, Tumor diameter, Lymphatic metastasis.

How to cite this article: Kubat M, Karabulut Z. Comparison of Tumor Volume Dependent Mean Diameter with the Longest Diameter and Tumor Volume in Assessing Axillary Lymph Nodes in Breast Cancer. J Coll Physicians Surg Pak 2020; 30(06):595-600 https://doi.org/10.29271/jcpsp.2020.06.595.

\section{INTRODUCTION}

Tumor size and axillary lymph node metastasis (ALNM) are the most important prognostic factors in breast cancer. ${ }^{1}$ Tumor lymph node-metastasis (TNM) staging system of American Joint Committee on Cancer (AJCC) used in breast cancer staging is one of the parameters used in the tumor's largest diameter (Dmax). ${ }^{2}$ Evaluation of tumor size in breast carcinoma is problematic due to its irregular shape. With the improved imaging methods, it has become quite easy to evaluate the three dimensions of the lesions. ${ }^{3}$

Correspondence to: Zülfikar Karabulut, Department of General Surgery, ALKU Alanya Training and Research Hospital, 07400, Alanya, Turkey

E-mail:zkarabulut101@gmail.com

Received: May 05, 2020; Revised: June 15, 2020;

Accepted: June 22, 2020

DOI: https://doi.org/10.29271/jcpsp.2020.06.595
Dmax, currently used in staging systems, was preferred during the years when one-dimensional imaging methods were frequently used because of its ease of use. However, in breast cancer cases, only one-third of tumors have a spherical or near-spherical shape. In ellipsoid or asymmetrical tumors that make up the majority, Dmax obtained by one-dimensional measurements cannotcompletely show the tumorburden. ${ }^{4}$

The main indicator of tumor burden is tumor volume (vTm) and its relationship with prognosis has been established in the literature. ${ }^{5}$ Disease management in numerous solid organ malignancies has made progress with regard to use of cross-sectional imaging and tumor measurement. ${ }^{6}$ However, existing literature and staging systems continue on one-dimensional parameters. ${ }^{2}$ Therefore, without losing the effectiveness of VTm in demonstrating tumor burden, a need for a parameter which is more closely related to vTm and compatible with literature and staging systems arises.

With the prominence of sentinel lymph node (SLN) evaluation in clinical practice, less invasive interventions have been performed compared to axillary lymph node dissection (ALND). 
Despite this, the majority of patients still encounter adverse conditions such as shoulder dysfunction, lymphedema and nerve damage. ${ }^{7}$ The fact that the results are negative in approximately $75 \%$ of SLN samples, shows that unnecessary risk is taken. ${ }^{8}$ Knowledge on the tumor characteristics, including the size of the tumor and the condition of the axillary lymph nodes before treatment, affects the treatment planning. Assessing the possibility of ALNM before treatment has been an important target for surgeons. In this way, unnecessary ALND can be avoided. There are various publications and various nomograms on this issue. ${ }^{9,10}$

In this study, the objective was to determine association of Rmax, Dmax and vTm parameters with ALNM positivity by determining the average diameter (Rmax) calculated from the tumor volume on the measured dimensions.

\section{METHODOLOGY}

Patients who underwent modified radical mastectomy (MRM) and breast-conserving surgery (lumpectomy) and axillary lymph node dissection due to invasive breast carcinoma at Ankara Training and Reseach Hospital, Turkey from January 2009 to December 2018 were included in this retrospectively planned descriptive study. Males and patients who underwent sentinel lymph node biopsy were excluded from the study. Patients who received neoadjuvant therapy, in situ cancer cases, recurrent cases, and those with multicentric and multifocal tumors were also excluded. After applying the exclusion criteria, the remaining 305 patients were included in the study.

The tumor size measurements obtained in the postoperative pathological evaluation results were recorded. Tumor sizes were obtained as a result of pathological examination and recorded as $a \geq b \geq c(a=D m a x)$. Number of metastatic lymph nodes and reactive lymph nodes was obtained from the pathological examination of axillary lymph node dissections and recorded in the system. Demographic, pathological, surgical data of the patients (age, gender, side, tumor type, type of surgery performed, location, lymphovascular invasion status, estrogen receptor positivity, progesterone receptor positivity, positivity of Her2/neu) were recorded for use in nomograms.

Approximate tumor volume was calculated from the obtained tumor specimen sizes $(a \geq b \geq c)$ using ellipsoid volume formula $[(a / 2) *(b / 2) *(c / 2) * \pi *(4 / 3)]$. From the sphere volume formula $\left[(\mathrm{R} / 2)^{3} * \pi^{*}(4 / 3)\right], \mathrm{R}$ max, which gives the same volume value, was reversely calculated $(R=R \max )$.

Rmax efficacy and usability were evaluated on two nomograms which are available on the internet and are applied most frequently owing to their ease of use. Nomograms were used to predict sentinel lymph node metastasis positivity, i.e. ALNM positivity. These nomograms included those that are used by the Memorial Sloan Kettering Cancer Center (MSKCC), the sentinel Iymphnode metastasis nomogram, ${ }^{9}$ and the breast cancer nomogram to predict positive sentinel lymph nodes, without neoadjuvant chemotherapy nomogram provided by MD Anderson Cancer Center (MDACC). ${ }^{10}$
Average, standard deviation, median, IQR, frequency and ratio values were used in the descriptive statistics of the data. The distribution of variables was measured by Kolmogorov-Smirnov test. In the analysis of quantitative independent data, MannWhitney test was used. In the analysis of qualitative independent data, Chi-square test was performed. Fischer test was used when Chi-square test conditions were not met. The effect level was investigated by the ROC curve. The effect level was investigated by univariate and multivariate logistic regression. SPSS 26.0 package programme was used in the analysis. The $p$-values less than 0.05 were regarded as significant.

All data collection and analyses were carried out with the approval of the Institutional Review Board of Alaaddin Keykubat University (approval number 26.10.2018/3-2) and the need for written informed consents was waived because of the low risk posed by this research. The study protocol confirmed to the ethical guidelines of the 1975 Declaration of Helsinki as reflected in approval by the Board.

\section{RESULTS}

After implementing patient selection criteria, the remaining 305 patients were included in the study. The mean age of the patients was $53.0 \pm 12.2$ years. The tumor was located in the right breast in $55.1 \%(n=168)$ of the patients. MRM was performed in $87.22 \%$ $(n=266)$ of the patients and $10.8 \%(n=33)$ of tumors were located in the upper inner quadrant. The mean number of lymph nodes examined in pathological specimens was 18.43 \pm 7.15 . The mean Dmax was $3.4 \pm 1.8 \mathrm{~cm}$, the mean of the vTm calculated using the ellipsoid formula was $17.9 \pm 35.9 \mathrm{~cm}^{3}$ and the average of the Rmax values calculated over the vTm values was $2.6 \pm 1.4 \mathrm{~cm}$.

Regarding tumor types, ductal $1(p=0.006)$ and mucinous $(p=0.023)$ carcinoma ratio wassignificantly higher in ALNM negative group, while ductal 3 tumor type ratio was significantly higher in the ALNM positive group ( $p=0.029)$. In ALNM positive and negative groups, Ductal $2(p=0.882)$, lobular $(p=0.773)$, medullary ( $p=0.700)$ tumor type ratios did not differ significantly (Table I). In ALNM positive group; Dmax, Rmax and vTm values were significantly higher ( $p^{<} 0.001$, Table I). The lymphovascular invasion rate was significantly higher in the ALNM positive group ( $p<0.001$ ). The estrogen receptor (ER) positivity rate and progesterone receptor (PR) positivity rate were significantly higher in the ALNM negative group ( $p=0.013$ and $p=0.024$ ) than the ALNM positive group (Tablel).

In the univariate model, tumor side $(p=0.043)$, ductal 1 $(p=0.008)$, ductal $3(p=0.030)$, mucinous carcinoma $(p=0.034)$ types and Dmax $(p<0.001), \operatorname{Rmax}(p<0.001)$, vTm $(p<0.001)$, lymphovascular invasion $(p<0.001)$, ER $(p=0.014), \quad P R$ $(p=0.024)$, number of total exemined lymph nodes $(p<0.001)$, the risk value calculated using Dmax with MSKCC nomogram $(p<0.001)$, the risk value calculated using Rmax with MSKCC nomogram ( $p<0.001)$, the risk value calculated using Dmax with MDACC nomogram $(p<0.001)$, the risk value calculated using Rmax with MDACC nomogram $(p<0.001)$ were observed to have significanteffectivenes 


\begin{tabular}{|c|c|c|c|c|c|}
\hline & \multicolumn{2}{|c|}{ ALNM (-) } & \multicolumn{2}{|c|}{ ALNM $(+)$} & \multirow[b]{2}{*}{$p$} \\
\hline & $n=133$ & $\%$ & $n=172$ & $\%$ & \\
\hline \multicolumn{6}{|l|}{ Tumor type } \\
\hline Ductal 1 & 18 & $13.5 \%$ & 8 & $4.7 \%$ & $0.006^{x^{2}}$ \\
\hline Ductal 2 & 70 & $52.6 \%$ & 92 & $53.5 \%$ & $0.882^{x^{2}}$ \\
\hline Ductal 3 & 29 & $21.8 \%$ & 57 & $33.1 \%$ & $0.029^{\mathrm{x}^{2}}$ \\
\hline Lobuler & 6 & $4.5 \%$ & 9 & $5.2 \%$ & $0.773^{x^{2}}$ \\
\hline Meduller & 2 & $1.5 \%$ & 4 & $2.3 \%$ & $0.700^{x^{2}}$ \\
\hline Musinous & 8 & $6.0 \%$ & 2 & $1.2 \%$ & $0.023^{x^{2}}$ \\
\hline \multicolumn{6}{|l|}{ Tumor side } \\
\hline Right & 82 & $61.7 \%$ & 86 & $50.0 \%$ & $0.042^{x^{2}}$ \\
\hline \multirow{2}{*}{\multicolumn{6}{|c|}{ Type of surgery }} \\
\hline & & & & & \\
\hline $\mathrm{LMP}+\mathrm{A} 18$ & $13.5 \%$ & 21 & $12.2 \%$ & $0.731^{x^{2}}$ & \\
\hline MRM & 115 & $86.5 \%$ & 151 & $87.8 \%$ & \\
\hline \multicolumn{6}{|l|}{ Upper inner quadrant } \\
\hline & 120 & $90.2 \%$ & 152 & $88.4 \%$ & $0.605^{x^{2}}$ \\
\hline & 13 & $9.8 \%$ & 20 & $11.6 \%$ & \\
\hline \multicolumn{6}{|l|}{ Lyphovascular invasion } \\
\hline & 106 & $79.7 \%$ & 36 & $20.9 \%$ & $<0.001^{x^{2}}$ \\
\hline & 27 & $20.3 \%$ & 136 & $79.1 \%$ & \\
\hline \multicolumn{6}{|l|}{ Estrogen receptor } \\
\hline & 27 & $20.3 \%$ & 57 & $33.1 \%$ & $0.013^{x^{2}}$ \\
\hline & 106 & $79.7 \%$ & 115 & $66.9 \%$ & \\
\hline \multicolumn{6}{|l|}{ Progesterone receptor } \\
\hline & 47 & $35.3 \%$ & 83 & $48.3 \%$ & $0.024^{x^{2}}$ \\
\hline \multirow{2}{*}{\multicolumn{6}{|c|}{$\stackrel{(+)}{\text { HER2 }}$}} \\
\hline & & & & & \\
\hline & 95 & $71.4 \%$ & 105 & $61.0 \%$ & $0.058^{x^{2}}$ \\
\hline$(+)$ & 38 & $28.6 \%$ & 67 & $39.0 \%$ & \\
\hline Avg \pm SD Median (IQR) & Avg \pm SD & Median (IQR) & Avg \pm SD & Median (IQR) & $p$ \\
\hline Dmax & $2.71 \pm 1.26$ & $2.50(1.5)$ & $3.98 \pm 2.00$ & $3.50(2.5)$ & $<0.001^{\mathrm{m}}$ \\
\hline$R \max$ & $2.00 \pm 1.02$ & $1.88(0.96)$ & $3.01 \pm 1.42$ & $2.86(1.51)$ & $<0.001^{\mathrm{m}}$ \\
\hline vTm & $8.46 \pm 20.65$ & $3.50(5.06)$ & $25.19 \pm 42.90$ & $12.21(19.98)$ & $<0.001^{\mathrm{m}}$ \\
\hline No. of MLN & $0.0 \pm 0.1$ & $0.0(0)$ & $6.6 \pm 7.0$ & $4.0(8)$ & $<0.001^{\mathrm{m}}$ \\
\hline No. of TELN & $16.5 \pm 6.3$ & $15.0(8)$ & $19.9 \pm 7.4$ & $18.5(12)$ & $<0.001^{\mathrm{m}}$ \\
\hline MSKCC (Dmax) Risk & $0.46 \pm 0.19$ & $0.42(0.25)$ & $0.70 \pm 0.19$ & $0.73(0.30)$ & $<0.001^{\mathrm{m}}$ \\
\hline MSKCC (Rmax) Risk & $0.38 \pm 0.18$ & $0.33(0.23)$ & $0.63 \pm 0.19$ & $0.65(0.26)$ & $<0.001^{\mathrm{m}}$ \\
\hline MDACC (Dmax) Risk & $0.30 \pm 0.16$ & $0.24(0.18)$ & $0.55 \pm 0.22$ & $0.57(0.33)$ & $<0.001^{\mathrm{m}}$ \\
\hline MDACC (Rmax) Risk & $0.25 \pm 0.14$ & $0.21(0.13)$ & $0.48 \pm 0.20$ & $0.50(0.30)$ & $<0.001^{\mathrm{m}}$ \\
\hline $\begin{array}{l}\text { mMann-whitney u test } /{ }^{R^{m}} \\
\text { Mastectomy; HER2 }=\text { Hun } \\
\text { (Dmax /Davg) Risk }=\text { Risk } \\
\text { Anderson Cancer Center }\end{array}$ & $\begin{array}{l}\text { t). ALNM } \\
\text { or Receptc } \\
\text { torial Sloa }\end{array}$ & $\begin{array}{l}\text { Ode Metast } \\
\text { Number of } \\
\text { Center non }\end{array}$ & $\begin{array}{l}\text { lpectomy +A } \\
\text { vode; No. of } \\
\text { X/Davg; MDA }\end{array}$ & $\begin{array}{l}\text { Node Dissect } \\
\text { ber of Total Ex } \\
\text { Davg) Risk }=R\end{array}$ & $\begin{array}{l}=\text { Modified Radical } \\
\text { mph Node; MSKCC } \\
\text { :alculated with MD }\end{array}$ \\
\hline
\end{tabular}

Table I: Differences between ALNM positive and negative groups.

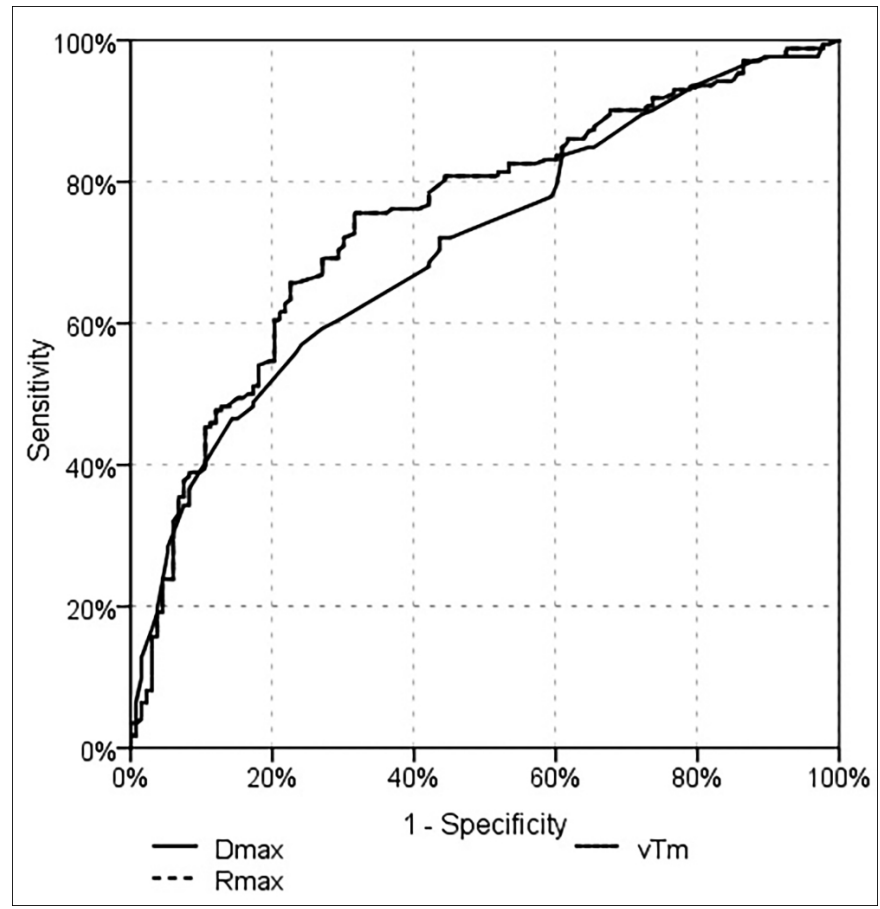

Figure 1: Evaluation of lymph node metastasis prediction efficiency of Dmax, vTm and Rmax with ROC Curve (The VTm and Rmax curves are superimposed).

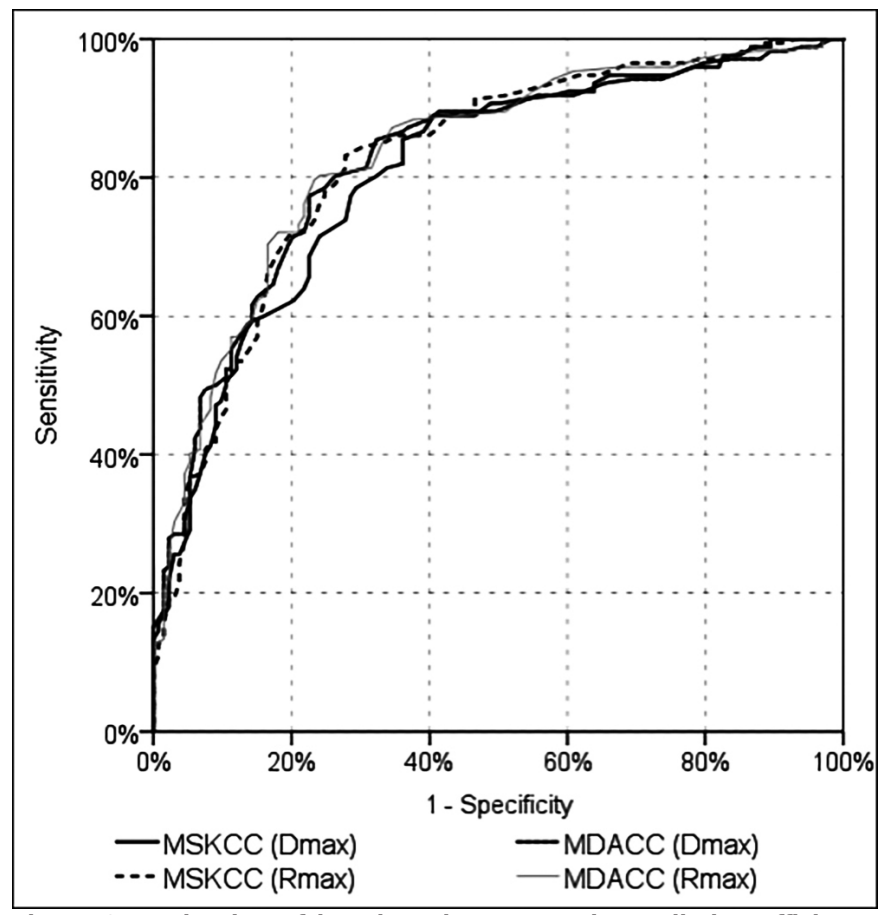

Figure 2: Evaluation of lymph node metastasis prediction efficiency of MSKCC and MDACC nomograms by ROC curve according to Dmax and Rmax parameters. 
In the multivariate reduced model, significant-independent efficacy of $R \max (p<0.001)$, lymphovascular invasion $(p<0.001)$, and number of the total lymph nodes was observed $(p=0.037)$ in differentiating the patients with and without lymph node metastasis. In differentiating the ALNM positive and negative patients, Dmax's significant [AUC = $\left.0.709(95 \% \mathrm{Cl}=0.652-0.766) p^{<} 0.001\right]$, Rmax's significant [AUC $\left.=0.748(95 \% \mathrm{Cl}=0.692-0.803) p^{<} 0.001\right]$ and VTm's significant [AUC $=0.748(95 \% \mathrm{Cl}=0.692-0.803) p^{<} 0.001$ ] efficacies were observed (Figure 1). In differentiating the ALNM positive and negative patients, following risk values were observed to have significant efficacy: the risk value calculated using Dmax with MSKCC nomogram [AUC $=0.810$ (95\% Cl $\left.=0.761-0.858) p^{<} 0.001\right)$, the risk value calculated using Rmax with the MSKCC nomogram [AUC = 0.825 (95\% $\left.\mathrm{Cl}=0.778-0.872) p^{<} 0.001\right]$, the risk value calculated using Dmax with the MDACC nomogram [AUC $=0.825(95 \% \mathrm{Cl}=$ $\left.0.778-0.872) p^{<} 0.001\right]$, the risk value calculated using the MDACC nomogram and $\mathrm{Rmax}[\mathrm{AUC}=0.833(95 \% \mathrm{Cl}=$ 0.787-0.879) $\left.p^{<} 0.001\right]$ (Figure 2).

\section{DISCUSSION}

One of the most important indicators of prognosis in breast cancer is presence or absence of axillary lymph node metastasis (ALNM). Survival rates decrease proportionally with increasing number of positive axillary lymph nodes. ${ }^{11}$ It is well known that tumor morphology affects ALNM positivity as well as tumor histology. Breast cancers are threedimensional masses, but few are spherical. In a study performed in 165 invasive carcinoma cases, Wapnir et al. found that only $3.6 \%$ of the cases were spherical. ${ }^{12}$

The largest diameter of the tumor (Dmax) is a parameter used in staging systems and in most of the studies conducted on this topic. As studies on breast cancer and other solid tumors show, predictive value of Dmax on lymph node presence and prognosis remains lower than tumor volume (vTm). ${ }^{12,13}$ Simonka et al. reported that the increase in vTm correlated with both sentinel lymph node positivity and higher prevalence of ALNM. ${ }^{14}$ In this study, of the groups with and without ALNM revealed that Dmax and VTm parameters were both effective parameters, however it was found that vTm was a more effective parameter in predicting the presence of lymph node which was consistent with the literature. This indicates that the current tumor load is more effective in evaluating the tumor volume as it is a threedimensional parameter. ${ }^{12}$ Despite this information, in their study on patients with rhabdomyosarcoma, Ferrari et al. reported that three-dimensional evaluations of tumor size did not provide better prognostic information compared to one-dimensional measurements. ${ }^{15}$ Similarly, Pyo et al. evaluated the relationship between one-dimensional and three-dimensional measurements of the tumor and lymph node metastasis in cases with early gastric cancer, and found that there was no difference. ${ }^{16}$ Histopathological features of different tumor types lead to differences in growth, invasion and variations between results.

However, using the vTm parameter instead of Dmax, which is used currently in evolution systems and literature, requires a radical change and this would cause confusion. ${ }^{2}$ The tumor diameter calculated on vTm (Rmax), which was evaluated in this study, is directly related to vTm. It is a good indicator of the calculated tumor burden and is easier to use than tumor volume. Its effectiveness in predicting the presence of lymph node was found to be the same as tumor volume. Since a one-dimensional result is obtained as a parameter, it will be the biggest advantage that it can be used in the existing cancer research, staging and treatment protocols without requiring a parameter change.

When the tumor mass increases, the difference between the sizes increases. They usually move away from the spherical shape and become asymmetrical. Wapnir et al. stated that the relationship between Dmax and vTm decreases as the lesion sizes increase. ${ }^{12}$ Hamza et al. reported that parameters calculated with the formula (equivalent in our study: vTm, Rmax) reduce the risks of overestimation and underestimation for outcomes ${ }^{17}$. In the present study, the difference between Dmax and Rmax increased as the lesion grew. When the relationship between ALMN and these parameters is examined, we concluded that as the lesion size increases, more overestimation of outcome occurs.

Nomograms are scales used to make predictions about a disease by combining multiple clinical variables and their intertwined relationships. The use of multiple variables provides a more precise and personalised estimate. ${ }^{18}$ In order for the nomogram to be employable, its effectiveness should be evaluated and the concordance index value should be at least $0.7 .^{19}$ Increased c-index value indicates that the efficacy is also enhanced. Dmax is one of the indispensable parameters in nomograms used to predict ALNM in breast cancer cases. ${ }^{9,10}$ In this study, Rmax was applied instead of Dmax in nomograms used worldwide in breast cancer cases and evaluated the result. Its validated using Rmax value instead of Dmax in both commonly used nomograms. When evaluated with the Roc curve and compared the areas under the curve, statistically, a significant increase was found in the efficacy of predicting the presence of ALNM of nomograms when Rmax was used as the parameter, instead of Dmax.

In this study, lymphovascular invasion and Rmax parameters were associated with ALNM in the multivariate model. In their study, An et al. showed that lymphovascular invasion and metabolic tumor burden were associated with ALNM. ${ }^{20}$ They reported that the metabolic tumor burden is an effective parameter especially in T2 and T3 stage breast cancer. 
The situation becomes more complex in multifocal/ multicentric (MF/MC) tumor cases that we have not included in our patient group. Dmax, which is the largest among the foci, is currently used for staging. ${ }^{2,21}$ When the relationship of ALNM with the largest diameter of the tumor is examined in cases with MF/MC breast cancer, an increase in ALNM is observed in MF/MC tumors compared to unifocal cancers because of the smaller foci that are not taken into account. ${ }^{22}$ Therefore, there are studies using parameters such as mean diameter (simple mean), total diameter (sum of the diameters of all foci) and total tumor volume in cases with MF/MC tumors. ${ }^{23}$ In their study, Djordjevic-Jovanovic et al. suggest that an ALNM positivity higher than predicted and expected in patients with MF/MC tumors is not due to the histology of their tumors but to the underestimation of the tumor load by the current staging system. ${ }^{24}$ In this study, the effectiveness of Rmax, was calculated, based on the total tumor volume, in unifocal tumors. The authors will be eagerly monitoring the further studies in which Rmax's effectiveness is examined in a large cohort series with $\mathrm{MF} / \mathrm{MC}$ tumors.

The most important limitation of this study is its retrospective design. Tumor sizes obtained from preoperative evaluation were not used. The most important reason for choosing tumor sizes obtained by evaluating pathology specimens is that results were definitive. In one study, $56 \%$ of the measurements obtained in preoperative evaluations were correlated with pathological results. Although there is no statistical significance between the mean dimensions between gross and microscopic size measurements, it has been shown to alter stage assessment in approximately $31 \%$ of cases. ${ }^{17}$ This does not constitute an obstacle for preoperative clinical use, and improvement in technique; and experience in radiological imaging will bring the results of preoperative and pathological measurements closer to each other. The fact that the tumor tissues, which form the basis of our study, are not properly shaped and emerge as one of our limitations. No tumor has a perfectly ellipsoid shape either. The tumor volume calculated with the ellipsoid formula has almost always been found to be an overestimation. ${ }^{4}$ More realistic volume calculations can be made with current imaging methods. ${ }^{20,25}$ However, this situation currently requires an extra hardware and increases the cost burden. In our hospital, advanced 3D and metabolic imaging methods are not used as standard methods. As 3D imaging becomes the standard method with the developing technology, volume calculations can be made directly by imaging methods, not indirectly as we do using the formula like ellipsoid etc.

\section{CONCLUSION}

When the relationships of Dmax and vTm with ALNM are compared in cases with breast cancer, the effectiveness of
vTm is higher. However, Rmax calculated on the volume related to VTm, can be used instead of Dmax. Rmax parameter used instead of Dmax, had higher relation with ALNM. In the nomogram samples still used in predicting ALNM, Rmax parameter was found to enhance the nomogram efficacies.

\section{ETHICAL APPROVAL:}

All data collection and analyses were carried out with the approval of the The Ethics Committee of Alaaddin Keykubat University (approval number 26.10.2018/3-2).

\section{PATIENTS' CONSENT:}

As the study was designed retrospectively, data was collected from clinical archive after ethical approval.

\section{CONFLICT OF INTEREST:}

Authors declared no conflict of interest.

\section{AUTHORS' CONTRIBUTION:}

MK: Study design, data collection, literature review, writing and statistical analysis.

ZK: Drafting of work, data collection, editing, final review and correspondence.

All authors have critically reviewed the manuscript.

\section{REFERENCES}

1. Carter $\mathrm{CL}$, Allen $\mathrm{C}$, Henson DE. Relation of tumor size, lymph node status, and survival in 24,740 breast cancer cases. Cancer 1989; 63(1):181-7.

2. Giuliano AE, Edge SB, Hortobagyi GN. Eighth edition of the ajcc cancer staging manual: Breast cancer. Ann Surg Oncol 2018; 25(7):1783-5.

3. Zhang S, Ding Y, Zhu Q, Wang C, Wu P, Dong J. Correlation factors analysis of breast cancer tumor volume doubling time measured by 3D-ultrasound. Med Sci Monit 2017; 23:3147.

4. Clarke G, Holloway C, Zubovits J, Nofech-Mozes S, Murray M, Liu K, et al. Three-dimensional tumor visualisation of invasive breast carcinomas using whole-mount serial section histopathology: Implications for tumor size assessment. Breast Cancer Res Treat 2019; 174(3): 669-77.

5. Hylton NM, Gatsonis CA, Rosen MA, Lehman CD, Newitt DC, Partridge SC, et al. Neoadjuvant chemotherapy for breast cancer: Functional tumor volume by MR imaging predicts recurrence-free survival-results from the ACRIN 6657/CALGB 150007 I-SPY 1 TRIAL. Radiology 2016; 279 (1):44-55.

6. Xie Hj, Zhang X, Mo YX, Long H, Rong Th, Su Xd. Tumor volume is better than diameter for predicting the prognosis of patients with early-stage non-small cell lung cancer. Ann Surg Oncol 2019; 26(8):2401-8.

7. Lorek A, Stojčev Z, Zarębski W, Kowalczyk M, Szyluk K. Analysis of postoperative complications after 303 sentinel lymph node identification procedures using the SentiMag ${ }^{\circledR}$ method in breast cancer patients. Med Sci Monitor 2019; 
25:3154.

8. Bilimoria KY, Bentrem DJ, Hansen NM, Bethke KP, Rademaker AW, Ko CY, et al. Comparison of sentinel lymph node biopsy alone and completion axillary lymph node dissection for node-positive breast cancer. J Clin Oncol 2009; 27(18):2946-53.

9. Bevilacqua JLB, Kattan MW, Fey JV, Cody III HS, Borgen PI, Van Zee KJ. Doctor, what are my chances of having a positive sentinel node? A validated nomogram for risk estimation. J Clin Oncol 2007; 25(24):3670-9.

10. Veerapong J, Boughey J, Mittendorf E, Harrell R, Bassett R, Ross M. A validated risk assessment of sentinel lymph node involvement in breast cancer patients. Ann Surg Oncol 2011; 18:S59.

11. Lakhani SR. WHO classification of tumours of the breast: International Agency for Research on Cancer 2012.

12. Wapnir IL, Wartenberg DE, Greco RS. Three dimensional staging of breast cancer. Breast cancer Res Trea 1996; 41(1):15-9.

13. Pennington TE, Thwin M, Sywak M, Delbridge L, Sidhu S. Sonographic volumetric assessment is a more accurate measure than maximum diameter alone in papillary thyroid cancer. J End Soc 2018; 2(11):1284-92.

14. Simonka Z, Anna M, Nagy A, Ormándi K, Rárosi F, Attila P, et al. The role of MRI based tumor volume definition in the surgical therapy of breast cancer. Eur J Surg Oncolo 2019; 45(2):e100.

15. Ferrari A, Miceli R, Meazza C, Casanova M, Favini F, Morosi $C$, et al. Comparison of the prognostic value of assessing tumor diameter versus tumor volume at diagnosis or in response to initial chemotherapy in rhabdomyosarcoma. J Clinical Oncol 2010; 28(8):1322-8.

16. Pyo JH, Byeon SJ, Lee $\mathrm{H}$, Min YW, Min BH, Lee JH, et al. Measurement of tumor volume is not superior to diameter for prediction of lymph node metastasis in early gastric cancer with minute submucosal invasion. Oncotarget 2017; 8(69): 113758.

17. Hamza A, Sakhi R, Alrajjal A, Ibrar W, Miller S, Salehi S, et al. Tumor size in breast carcinoma: Gross measurement is important! Int J Surg Pathol 2018; 26(6):494-9.

18. Balachandran VP, Gonen M, Smith JJ, DeMatteo RP. Nomograms in oncology: More than meets the eye. lancet Oncol 2015; 16(4):e173-e80.

19. Kawai K, Sunami E, Yamaguchi H, Ishihara S, Kazama S, Nozawa $\mathrm{H}$, et al. Nomograms for colorectal cancer: A systematic review. World J Gastro 2015; 21(41):11877.

20. An YS, Kang DK, Jung Y, Kim TH. Volume-based metabolic parameter of breast cancer on preoperative 18F-FDG PET/CT could predict axillary lymph node metastasis. Med 2017; 96(45):e8557.

21. Gannon LM, Cotter MB, Quinn CM. The classification of invasive carcinoma of the breast. Expert Rev Anticancer Ther 2013; 13(8):941-54.

22. Weissenbacher TM, Zschage M, Janni W, Jeschke U, Dimpfl $\mathrm{T}$, Mayr D, et al. Multicentric and multifocal versus unifocal breast cancer: Is the tumor-node-metastasis classification justified? Breast Cancer Res Treat 2010; 122(1):27-34.

23. Boros M, Moldovan C, Varlam CM, Podoleanu C, Georgescu $\mathrm{R}$, Stolnicu S. Which is the best method to measure the size in multiple breast carcinoma in correlation with impact on prognosis? A retrospective study of 418 cases. Int J Clin Exp Med 2016; 9(2):3489-96.

24. Djordjevic Jovanovic L, Karanikolic A, Bojic T, Pesic I, Djordjevic M, Marinkovic M. Characteristics and outcomes of patients with multifocal/multicentric and unifocal breast cancer. J BUON 2017; 22(3):652-7.

25. Marinelli B, Espinet Col C, Ulaner GA, McArthur HL, Gonen $M$, Jochelson $M$, et al. Prognostic value of FDG PET/CT-based metabolic tumor volumes in metastatic triple negative breast cancer patients. Am J Nucl Med Mol Imaging 2016; 6(2):120. 